deux autres langues en bas du texte explicatif résulte simplement de l'ordre alphabétique. Pour les mots allemands, il faut observer

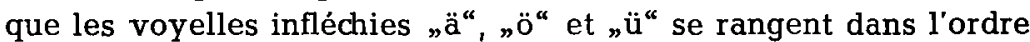
alphabétique sous "ae", „oe" et "ue".

Les flèches $(\rightarrow$ ) dans le texte des définitions renvoient à d'autres acceptions relatives au même sujet et que l'on aura intérêt à consulter pour une meilleure compréhension du terme recherché. L'acception n'est pas répétée dans le texte: elle est remplacée par un tilde ( ) .

Dans les articles concernant les différents pays, l'énumération des partis et des journaux n'est pas exhaustive; elle se limite aux groupes et titres essentiels. Les grandes organisations internationales sont reprises, dans l'ordre alphabétique, aussi bien sous leur appellation complète que sous leur abréviation usuelle. Généralement, les auteurs n'ont employé dans les textes que des abréviations courantes (voir le registre suivant).

L'annexe trilingue, comportant les graphiques des organismes mondiaux importants ainsi qu'une carte de l'Afrique avec les nouvelles frontières politiques sur ce continent, permettra sans doute au lecteur de se retrouver plus facilement dans la complexité des rapports internationaux. Les notes au bas des textes explicatifs renvoient à la page de l'annexe dans la langue correspondante.

\title{
Redaktionelle Hinweise
}

Für den sinnvollen Gebrauch des vorliegenden Wörterbuchs - und um alle in ihm enthaltenen Möglichkeiten voll ausnutzen zu können - sollte der Benutzer folgendes berücksichtigen:

Das Wörterbuch bringt die rund 14000 Stichworte in deutsch, englisch und französisch in einem integrierten Alphabet.

Das Auffinden der Stichworte wird durch Kolumnentitel am Kopf der Seiten erleichtert. Hinter jedem Stichwort (Fettsatz) folgen die knapp gehaltenen Erläuterungen bzw. Definitionen des Begriffes. Sie wurden thematisch auf den politisch-wirtschaftlichen Bereich begrenzt. Auf Hinweise auf die Bedeutung eines Stichwortes auf anderen Sachgebieten wurde verzichtet. 
Am Fuß eines jeden Stichwortes finden sich die fremdsprachlichen Hinweise (Kursiv), d. h. die Stichwort-Ubersetzungen unter denen man die gleichen Begriffe jeweils in den anderen beiden Sprachen findet. Sofern Stichworte in zwei oder allen drei Sprachen buchstabengleich sind, ist auf ihre zwei- bzw, dreifache Aufführung verzichtet worden. In diesen Fällen steht das Stichwort - auch in bezug auf die Groß- und Kleinschreibung - wie in seiner Ursprungssprache üblich. Die beiden anderen Sprachen werden mit einem Strich (-) eingeleitet. Dieses Verfahren ist aus Gründen der Einheitlichkeit auch in den Fällen beibehalten worden, in denen das durch einen Strich ersetzte deutsche Stichwort an sich großgeschrieben werden müßte.

Bei der Festlegung, in welcher Sprache Stichworte erscheinen sollten, die weder aus dem Deutschen, Englischen oder Französischen stammen, ist wegen seiner großen Verbreitung dem Englischen der Vorzug eingeräumt worden. Die Reihenfolge der fremdsprachlichen Fußnoten ergab sich zwingend aus dem Alphabet der jeweiligen Sprache des Stichwortes. Für die deutschen Stichworte ist zusätzlich

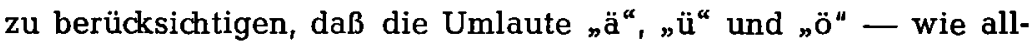
gemein üblich - im Alphabet in "ae", "ue" und "oe" aufgelöst worden sind.

Pfeile $(\rightarrow)$ in den Definitionen verweisen auf andere Stichworte, die im Sachzusammenhang stehen und deren Definition zum besseren Verständnis beitragen. Bei der Wiederholung des Stichwortes im Text der Definitionen steht eine Tilde $(\sim)$.

Die in den Erläuterungen der Länder-Stichworte aufgeführten Hinweise auf Parteien und Zeitungen wurden auf wesentlich Erscheinendes beschränkt. Sie können naturgemäß keinen vollständigen Katalog darstellen. Die großen internationalen Organisationen sind bewußt sowohl unter ihrem vollen Namen als auch unter den gebräuchlichen Abkürzungen aufgeführt. Allgemein wurde, wie das nachfolgende Abkürzungsverzeichnis aufweist, nur auf bekannte Abkürzungen zurückgegriffen.

Der ebenfalls dreisprachige Anhang mit den graphischen Schau* bildern wichtiger Organisationen sowie die Afrika-Karte mit den neuen politischen Grenzen auf diesem Kontinent ist sicher geeignet, die häufig verwirrenden Zusammenhänge und Beziehungen optisch klarer erfassen zu helfen. Fußnoten bei den Stichworten verweisen auf den entsprechenden Anhang in der jeweiligen Sprache. 